\title{
Osteoporosis Prevalence and Correlates in Uncomplicated Type 2 Diabetes Mellitus
}

\author{
Amit Nachankar \\ Department of Endocrinology, Army Hospital (R and R), New Delhi, India.
}

Corresponding Author: Amit Nachankar, Department of Endocrinology, Army Hospital (R and R), New Delhi, India.

\section{Received Date: 20 October 2021 | Accepted Date: 18 November 2021 | Published Date: 24 November 2021}

Citation: Amit Nachankar, Osteoporosis Prevalence and Correlates in Uncomplicated Type 2 Diabetes Mellitus. J. Diabetes and Islet Biology, 3(3); DOI: $10.31579 / 2641-8975 / 028$

Copyright: (c) 2021 Amit Nachankar, This is an open access article distributed under the Creative Commons Attribution License, which permits unrestricted use, distribution, and reproduction in any medium, provided the original work is properly cited.

\begin{abstract}
Type 2 Diabetes Mellitus (T2DM) is a common metabolic disorder at a pandemic proportion at present. Often T2DM is associated with microvascular (diabetic nephropathy, neuropathy, and retinopathy) and macrovascular complications (coronary artery disease, peripheral arterial disease, and stroke). Additionally diabetic osteopathy is a significant comorbidity of T2DM and is characterized by micro architectural changes that decrease bone quality leading to an increased risk of fragility fracture.
\end{abstract}

Keywords: osteoporosis prevalence; type 2 diabetes mellitus; metabolic disorder

\section{Introduction:}

Type 2 Diabetes Mellitus (T2DM) is a common metabolic disorder at a pandemic proportion at present. Often T2DM is associated with microvascular (diabetic nephropathy, neuropathy, and retinopathy) and macrovascular complications (coronary artery disease, peripheral arterial disease, and stroke) [1]. Additionally diabetic osteopathy is a significant comorbidity of T2DM and is characterized by micro architectural changes that decrease bone quality leading to an increased risk of fragility fracture. [2]

Individuals with T2DM are often associated with a reduction of bone strength which might not be reflected in the measurement of bone mineral density (BMD). They show relatively reduced bone formation, osteoblast dysfunction, and low bone turnover. The low bone turnover has deleterious effects on bone health. Insulin deficiency decreases bone growth and turnover by decreased osteoblast recruitment. In advanced T2DM, insulin deficiency could impair bone homeostasis through dysregulation of the growth hormone-IGF-1 axis. Pooled data has shown that bone turnover markers (BTM) such as osteocalcin, procollagen type 1 amino terminal pro-peptide and bone resorption markers such as $\mathrm{C}$ terminal cross-linked telopeptide were significantly lower among those with T2DM and did not necessarily correlate with glucose level. So T2DM should be considered a state of low bone turnover, perhaps driven by increased serum levels of sclerostin and osteoprotegerin which inhibit osteoblast and osteoclast differentiation respectively.

Strotmeyer et al [3] have shown increased BMD in T2DM due to associated obesity but also increased risk of fragility fractures due to altered bone microarchitecture. Dutta et al [4] have showed decreased BMD in T2DM. Some studies have shown association of poor glycemic control with increased osteoporosis and fragility fractures whereas others have shown no effect of glycemic control on fragility fractures in T2DM. Many studies have shown osteopenic effects of certain antidiabetic drugs (thiazolidinediones, SGLT 2 inhibitors and insulin) on increased fragility fracture prevalence in T2DM. Hence, the present study was conducted with the aim to evaluate the prevalence of osteoporosis in T2DM using the BMD by dual Xray absorptiometry (DXA) and its correlation with various factors in uncomplicated T2DM population.

\section{Materials and methods:}

This cross-sectional observational study was conducted at a multidisciplinary tertiary care hospital in North India involving 100 T2DM patients attending medicine/endocrinology outpatient department. They were enrolled during the study period of 1.5 year (From Mar 2019 to Sep 2020). The inclusion criteria was T2DM patients of age 50 years and above, either gender, new or known T2DM. The exclusion criteria were: Patients diagnosed with diabetes-associated microvascular and macrovascular complications, chronic smokers, chronic kidney disease, thyroid/parathyroid disorders, obvious bone and mineral disorders, chronic pancreatitis or postpancreatectomy, systemic inflammatory disorders, malabsorption syndromes, malignancy, or those receiving corticosteroids, immunosuppressants, anticonvulsants, thiazolidinediones or insulin; chronic debility or bedridden patients and those unwilling to participate in study.

After ethical approval and written consent of the patients, all the patients falling in sampling frame were enrolled. Demographic information was obtained from all the patients. Clinical history, duration of diabetes, treatment history and current medication profile was noted. All underwent anthropometric assessment and body mass index (BMI) was calculated. A detailed clinical examination was carried out. Blood samples were collected in fasting and 2 hour post prandial from all the patients and were 
analyzed for fasting blood glucose (FBG) \& post prandial blood glucose (PPBG), HbA1c, serum calcium, phosphorus, total protein, albumin and Vitamin D levels. All the patients underwent BMD-DXA evaluation by Hologic machine at two sites - spine and hip. For each site, calibration was performed before scans in every patient. Proper positioning was ascertained before and during the scans. The same trained technician performed DXA scans in all patients. T-scores were calculated. As recommended by the World Health Organization a T-score of 2.5 or lower was considered as osteoporosis at either site while T-score between -1.0 and -2.4 was considered as osteopenia. Data was collected on a proforma and was later entered into computer using Microsoft Excel software.

\section{Statistical Methods:}

Data was analyzed using Statistical Package for Social Sciences, version 21.0. Qualitative data has been represented as numbers and percentages

while quantitative/ continuous data has been represented as mean \pm standard deviation. Analysis of variance and chi-square test were used to compare the data. A 'p' value less than 0.05 was considered as statistically significant.

\section{Results:}

The baseline profile of the subjects in the study is as in table 1 . The age of patients ranged from 50-80 years, with a mean age of 59.39 \pm 7.01 years. The mean BMI of patients was $25.94 \pm 9.97 \mathrm{~kg} / \mathrm{m}^{2}$ and the BMI ranged from $16.44-34.02 \mathrm{~kg} / \mathrm{m}^{2}$. There were $40 \%$ overweight and $16 \%$ obese patients with $2 \%$ underweight. The duration of T2DM ranged from 1 to 15 years with mean of $6.21 \pm 2.56$ years. All were taking metformin for glycaemia control and a majority $(75.0 \%)$ of them were also taking Sulfonylureas. Only a few (28.0\%) were taking DPP4 inhibitors. The only comorbidity of primary hypertension was present in $23(23.0 \%)$ patients. The average fasting glucose of

\begin{tabular}{|c|c|c|c|c|c|}
\hline \multirow[t]{2}{*}{ SN } & \multirow[t]{2}{*}{ Parameter } & \multicolumn{4}{|c|}{ Statistics } \\
\hline & & Min. & Max. & Mean & SD \\
\hline 1 & Age (in years) & 50.0 & 80.0 & 59.39 & 7.01 \\
\hline 2 & BMI $\left(\mathrm{kg} / \mathrm{m}^{2}\right)$ & 16.44 & 34.02 & 25.94 & 9.97 \\
\hline 3 & Waist Circumference & 82 & 117 & 96.73 & 6.61 \\
\hline 4 & Systolic Blood Pressure (mmHg) & 110 & 152 & 127.34 & 7.22 \\
\hline \multirow[t]{2}{*}{5} & Diastolic Blood Pressure $(\mathrm{mmHg})$ & 62 & 90 & 76.89 & 5.86 \\
\hline & & \multicolumn{2}{|l|}{ No. } & \multicolumn{2}{|l|}{$\%$} \\
\hline \multirow[t]{3}{*}{6} & \multicolumn{5}{|l|}{ Gender } \\
\hline & Female & \multicolumn{2}{|l|}{68} & \multicolumn{2}{|l|}{68.0} \\
\hline & Male & \multicolumn{2}{|l|}{32} & \multicolumn{2}{|l|}{32.0} \\
\hline
\end{tabular}

Table 1: Distribution of study population at baseline $(N=100)$

$132 \mathrm{mg} / \mathrm{dl}$ and postprandial glucose was $182 \mathrm{mg} / \mathrm{dl}$ in the study population with average HbA1c 7.7\%. Distribution of Study Population according to BMD at different sites is shown in figure 1 . Overall $47 \%$ patients had
Osteopenia and 39\% had Osteoporosis. At Lumbar Spine, $43 \%$ patients had osteopenia and $35 \%$ had osteoporosis. Similarly at Hip, $54.0 \%$ had Osteopenia and only $19 \%$ had osteoporosis.

$\square$ Normal $\square$ Osteopenia $\square$ Osteoporosis

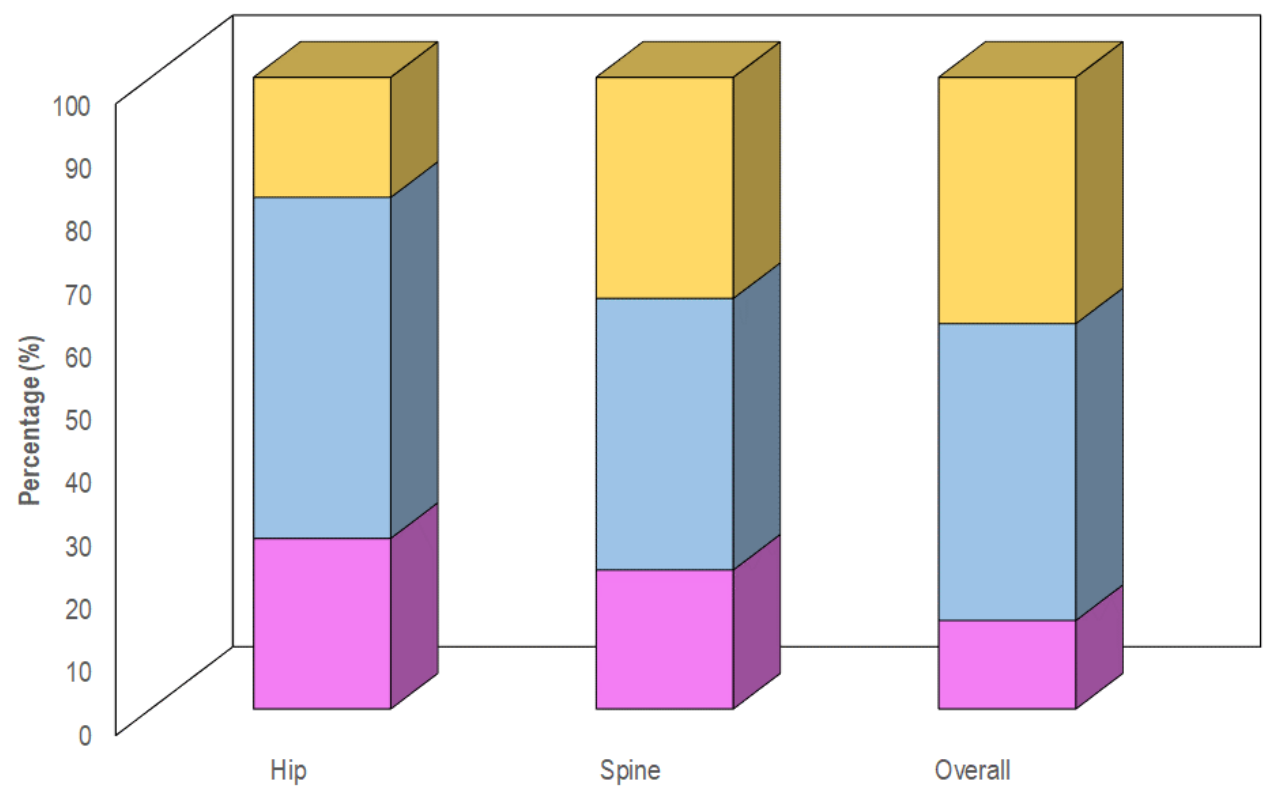

Figure 1: Distribution of Study Population as per BMD at different sites

For BMD findings, mean age of patients with osteoporosis $(61.26 \pm 6.92$ years) was higher as compared to cases with osteopenia $(59.15 \pm 7.20$ years) or normal BMD (55.0 \pm 4.42 years) and this difference was statistically significant $(\mathrm{p}=0.014)$. A higher proportion of females had osteopenia (68.1\%) and osteoporosis $(84.6 \%)$ as compared to males (osteopenia:31.9\%; osteoporosis:15.4\%) and this difference was statistically significant $(\mathrm{p}<0.001)$. However no significant association between severity of osteoporosis with waist circumference, BMI and nutritional status was found. No significant association between severity of osteoporosis with duration of diabetes was found $(\mathrm{p}=0.301)$. 
The mean FBG in osteoporosis patient $(122.49 \pm 26.43 \mathrm{mg} / \mathrm{dl})$ was lower than in osteopenia $(140.13 \pm 53.07 \mathrm{mg} / \mathrm{dl})$ or normal BMD $(130.93 \pm 27.95 \mathrm{mg} / \mathrm{dl})$. The mean PPBG in osteoporosis patient $(174.05 \pm 43.96 \mathrm{mg} / \mathrm{dl})$ was lower than in osteopenia $(190.36 \pm 71.57 \mathrm{mg} / \mathrm{dl})$ or normal BMD $(178.64 \pm 37.54 \mathrm{mg} / \mathrm{dl})$. The mean HbA1c in osteoporosis patients $(7.62 \pm 1.48 \%)$ was lower than in normal BMD $(7.52 \pm 5.02 \%)$ with osteopenia patients had HbAlc of $7.52 \pm 1.65 \%$. None of these showed any significant difference as in Table 2 .

\begin{tabular}{|l|l|l|l|l|l|l|l|l|l|l|}
\hline SN & Blood Sugar Levels & \multicolumn{2}{l|l|l|l|}{ Osteopenia $(\mathbf{n}=47)$} & \multicolumn{3}{l|}{ Osteoporosis $(\mathbf{n}=39)$} & \multicolumn{2}{l|}{ ANOVA } \\
\cline { 3 - 12 } & & Mean & SD & Mean & SD & Mean & SD & 'F' & p \\
\hline 1 & Fasting Blood Sugar (mg/dl) & 130.93 & 27.95 & 140.13 & 53.07 & 122.49 & 26.43 & 1.940 & 0.149 \\
\hline 2 & $\begin{array}{l}\text { Post-Prandial Blood Sugar } \\
\text { mg/dl) }\end{array}$ & 178.64 & 37.54 & 190.36 & 71.57 & 174.05 & 43.96 & 0.873 & 0.421 \\
\hline 3 & HbA1C (\%) & 8.65 & 5.02 & 7.52 & 1.65 & 7.62 & 1.48 & 1.299 & 0.277 \\
\hline
\end{tabular}

Table 2: Association of Severity of Overall BMD with Glycemic status

Normal BMD cases had a higher mean vitamin-D levels (32.68 \pm 17.90 $\mathrm{ng} / \mathrm{ml})$ as compared to osteopenia cases $(29.20 \pm 13.27 \mathrm{ng} / \mathrm{ml})$ or osteoporosis cases $(22.64 \pm 15.52 \mathrm{ng} / \mathrm{ml})$. (Table 3) This difference was found to be statistically significant $(\mathrm{p}=0.044)$. However, no significant association between severity of osteoporosis with calcium levels was found $(\mathrm{p}=0.226)$. Conversely majority $(46.2 \%)$ of osteoporosis cases had Vitamin-D deficiency, followed by insufficient Vitamin-D in $35.9 \%$. Only very few (17.9\%) osteoporosis cases had normal

\begin{tabular}{|l|l|l|l|l|l|l|l|l|l|}
\hline SN & Laboratory Parameters & \multicolumn{3}{l|}{ Normal $(\mathbf{n}=14)$} & \multicolumn{3}{l|}{ Osteopenia $(\mathbf{n}=47)$} & \multicolumn{3}{l|}{ Osteoporosis $(\mathbf{n}=39)$} & \multicolumn{2}{l|}{ ANOVA } \\
\cline { 3 - 10 } & & Mean & SD & Mean & SD & Mean & SD & 'F' & P \\
\hline 1 & S. Vitamin- D (ng/ml) & 32.68 & 17.90 & 29.20 & 13.27 & 22.64 & 15.52 & 3.215 & 0.044 \\
\hline 2 & S. Calcium (mg/dl) & 9.47 & 0.83 & 9.35 & 0.76 & 9.09 & 0.92 & 1.511 & 0.226 \\
\hline
\end{tabular}

Table 3: Association of BMD with Serum vitamin D and serum calcium

Vitamin D levels. However, higher proportions of osteopenia (48.9\%) and normal BMD cases $(57.1 \%)$ had normal vitamin D levels. Further, only 3 (21.4\%) normal BMD cases had vitamin D deficiency or insufficiency. Similarly $12(25.5 \%)$ osteopenia cases had vitamin D deficiency or insufficiency. This difference was statistically significant $(\mathrm{p}=0.044)$.

A majority (46.2\%) of osteoporosis cases had Vitamin-D deficiency, followed by insufficiency (35.9\%). Only $20 \%$ osteoporosis cases had normal vitamin D. However in osteopenia cases, higher proportions (48.9\%) had normal vitamin D, followed by $25.6 \%$ each having insufficiency or vitamin D deficiency. Similarly in normal BMD, majority (57.1\%) had normal vitamin $\mathrm{D}$, followed by $21.4 \%$ each having vitamin $\mathrm{D}$ deficiency or insufficiency. This difference was statistically significant $(\mathrm{p}=0.022)$. (Figure 2)

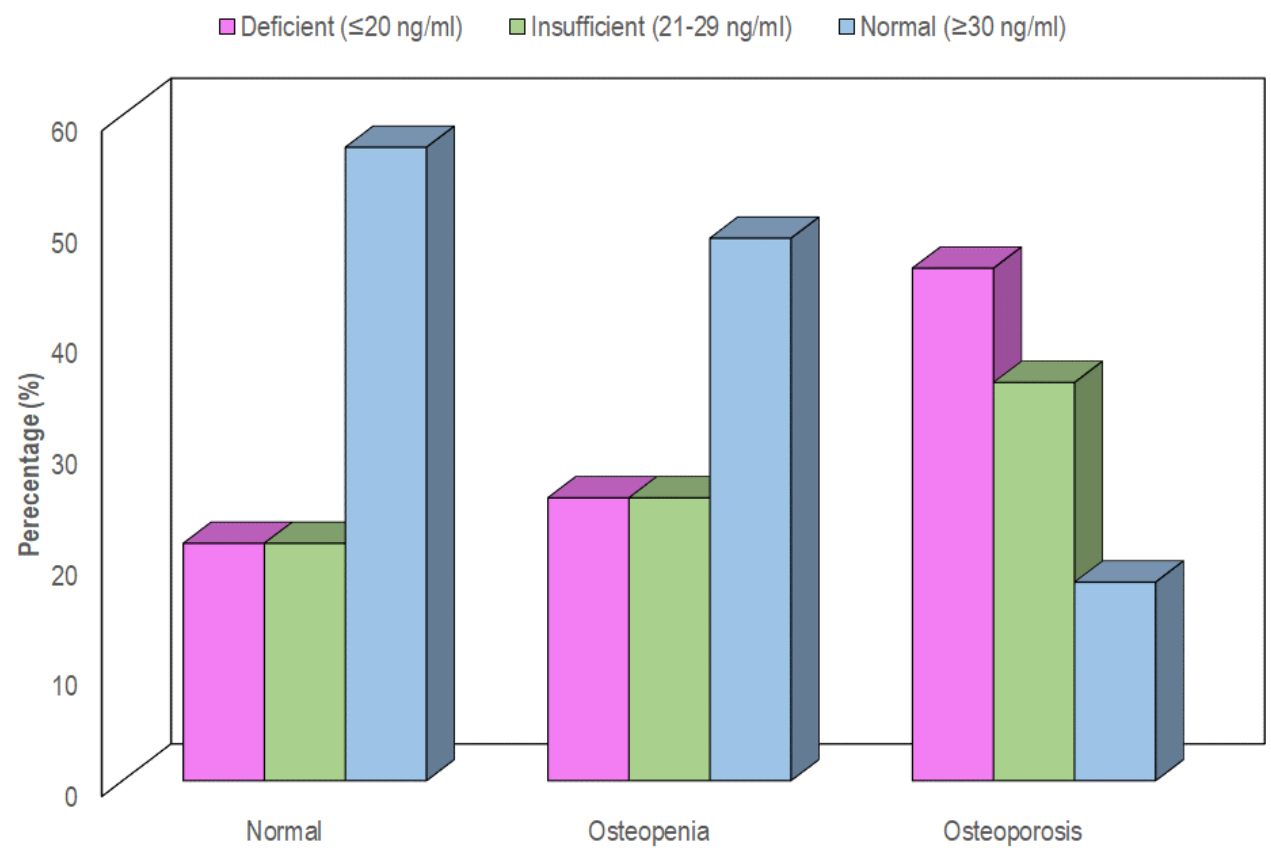

Fig 2: Association of Serum Vitamin D status with BMD 


\section{Discussion:}

Our study had a mean age of $59.39 \pm 7.01$ years similar to Agrawal and Sharma [5] whose study on 200 males aged 50 years or more reported mean age of $62.61 \pm 7.64$ years. A cross-sectional study by Sharma et al. [6] evaluated osteoporosis in $200 \mathrm{~T} 2 \mathrm{DM}$ patients and reported a mean age of $64.5 \pm 7.0$ years. Similar study by Asokan et al. [7] had mean age 56years in T2DM patients. Our study has 39\% prevalence of osteoporosis (with $47 \%$ osteopenia) thereby showing highly compromised BMD in T2DM. Compromised BMD has been reported to be quite high among T2DM in different studies from across different parts of India. Table 4 shows the profile of some of the contemporary studies from India studying the prevalence of osteopenia and osteoporosis in T2DM and compares them with our study. Low prevalence rate of

\begin{tabular}{|c|c|c|c|c|}
\hline SN & Author (Year) & Sample size and characteristics & Osteopenia & Osteoporosis \\
\hline 1. & $\begin{array}{l}\text { Kamalanathan et al. } \\
(2014)^{8}\end{array}$ & $\begin{array}{l}194 \\
(50 \% \text { males), Mean age } 42.9 \text { yrs - Males; } 39.3 \\
\text { yrs - females }\end{array}$ & \multicolumn{2}{|c|}{ Low $\mathrm{BMD}=19.5 \%$} \\
\hline 2. & Asokan et al. $(2017)^{7}$ & $\begin{array}{l}75 \\
\text { (Mean age } 56.17 \text { yrs), } \\
\text { Gender profile not clarified }\end{array}$ & $46.7 \%$ & $12.0 \%$ \\
\hline 3. & Sharma et al. $(2017)^{6}$ & $\begin{array}{l}200 \\
\text { (Mean age } 64.5 \mathrm{yrs}, \\
51.5 \% \text { males) }\end{array}$ & $51.5 \%$ & $35.5 \%$ \\
\hline 4. & Prakash et al. $(2017)^{10}$ & $\begin{array}{l}96 \\
\text { (Mean age } 62 \text { yrs, } \\
52.1 \% \text { males) }\end{array}$ & $26.0 \%$ & $43.8 \%$ \\
\hline 5. & $\begin{array}{l}\text { Thakur and } \quad \text { Dash } \\
(2018)^{13}\end{array}$ & $\begin{array}{l}60 \\
\text { (Mean age } 58.3 \mathrm{yrs} ; \\
56.7 \% \text { males) }\end{array}$ & $35.0 \%$ & $18.3 \%$ \\
\hline 6. & Reema et al. $(2020)^{11}$ & $\begin{array}{l}200 \\
\text { (Mean age 56.13 yrs, } \\
56.5 \% \text { females; Rural patients) }\end{array}$ & $29 \%$ & $53 \%$ \\
\hline 7. & Present study (2020) & $\begin{array}{l}100 \\
\text { (Mean age 59.39yrs; } \\
68 \% \text { females) }\end{array}$ & $47 \%$ & $39 \%$ \\
\hline
\end{tabular}

Table 4: Prevalence of osteopenia and osteoporosis in type 2 DM patients in different contemporary studies from India

low BMD (19.5\%) in the study of Kamalanathan et al. [8] could be primarily due to much younger patients (mean age 42.9 years for males and 39.3 years for females) as compared to other studies showing mean age 55-65 years at increased risk of osteoporosis. $\left[{ }^{9]}\right.$ However, most of the previous studies show a high prevalence of osteopenia and osteoporosis in T2DM.

Our study had relatively more osteopenia (47\%) patients than osteoporosis (39\%) which is in agreement with the observation of Sharma et al. ${ }^{6}$ who also had higher number of osteopenia (51.5\%) patients than osteoporosis $(35.5 \%)$. They had an overall prevalence of osteopenia and osteoporosis close to ours ( $87 \%$ vs $86 \%$ in our study). Though Prakash et al.[10] also reported the prevalence of osteopenia and osteoporosis to be quite high (69.8\%), however, in their study the prevalence of osteoporosis was higher $(43.8 \%)$ as compared to osteopenia (26\%). Reema et al. ${ }^{11}$ reported the overall prevalence of osteopenia and osteoporosis to be close to our study ( $82 \%$ vs $86 \%$ in our study), yet in their study proportion of osteoporosis was much higher $(53 \%)$ than our study (39\%) whereas proportion of those with osteopenia was much lower (29\%) than our study (47\%). These finding suggest that although, there is a high prevalence of osteopenia and osteoporosis in T2DM patients yet the pattern and spectrum of these BMD abnormalities shows a considerable variance. These findings imply that apart from T2DM other factors also play a role in BMD.

In our study, the osteoporosis prevalence at Lumbar spine (35\%) was higher than at hip (19\%). These findings are in agreement with the observations of Prakash et al. [10] who also found both prevalence of osteoporosis to be higher at Lumbar spine (39.6\%) as compared to hip (20.8\%). Sharma et al. [6] also observed that prevalence of osteoporosis at spine was $33.5 \%$ whereas at hip it was $13.5 \%$. A similar observation was also made by Kamalnathan et al.[8] who reported mean Z-scores for
BMD at spine to be lower as compared to that at hip. However, LeidigBruckner et al. ${ }^{12}$ reported the greater prevalence of osteoporosis at hip (13\% men and $21.9 \%$ women) than at spine $(6.1 \%$ men and $9.4 \%$ women). The difference could probably be owing to difference in occupation and lifestyle pattern of Asians as compared to western population.

Our study did not find a significant association of BMD with glycemic status i.e. FBG and PPBG levels or $\mathrm{HbA}_{1 c}$. Association of BMD with glycemic status are controversial. In a recent report Thakur and Dash [13] found mean $\mathrm{HbA}_{1 \mathrm{c}}$ of patients with low BMD to be significantly lower as compared to those having high BMD, thus showing good glycemic control to be associated with an increased risk of BMD loss. Conversely, they observed mean FBG of those with low BMD to be higher as compared to those having high $\mathrm{BMD}$, thus painting just a reverse picture as observed for $\mathrm{HbA}_{1 \mathrm{c}}$. Mobini et al.[14] in a study among T2DM women also found comparable $\mathrm{HbA}_{1 \mathrm{c}}$ levels between women with and without osteoporosis. Dutta et al. [4] also did not find a significant association between BMD and $\mathrm{HbA}_{1 \mathrm{c}}$ levels. In fact, the observation made by Kamalanathan et al.[8] ruled out any effect of glycemic status on BMD who in a case-control study did not find a significant difference in BMD of Indian T2DM patients as compared to healthy volunteers. Asokan et al.[7] also did not find a significant difference in BMD of T2DM cases as compared to controls, and thus ruled out an association of level of glycemic control with BMD. In our study, the non-existence of a relationship between level of glycemic control and BMD also showed that hyperglycemia per se cannot be the sole factor being responsible for BMD loss in T2DM.

In our study, age was found to be significantly associated with severity of osteoporosis. Moreover, the increased loss of BMD was observed with increasing age. These findings confirm that age continues to be one of the 
deterministic factors in BMD loss in T2DM as well. The relationship between age and BMD loss is governed by two factors. First, with advancing age there is an imbalance between resorption and formation (resorption higher than formation), thereby increased BMD loss. Secondly, increasing age results in degenerative changes thereby decreasing physical activity [9]. Reema et al.[11] in their study observed increasing age to be associated with increased odds of BMD loss in both univariate as well as multivariate analysis. Prakash et al.[10] also observed a positive correlation between BMD loss with advancing age. As such, the association between advancing age and BMD loss is a universal phenomenon seen in all the populations irrespective of T2DM status $\left[{ }^{15}, 16,17\right]$.

Our study did not find a significant association of waist circumference or BMI with BMD loss. Our observations are in contradiction with the observations of Leidig-Bruckner et al. [12] and Oei et al. [ ${ }^{18}$ ] where BMD was positively correlated with BMI. Reema et al.[11] and Prakash et al. [10]also found a protective effect of increased BMI against BMD loss. However, in our study, no such protective effect of BMI could be seen.

We also could not find a significant association between duration of diabetes and BMD status. However, a number of studies found duration of diabetes to be significantly associated with BMD loss [10,11,13,19]. One of the reasons could be primarily the present study had only few cases with T2DM duration >10 years (mean duration of T2DM 6.21 years). Compared to our study, Prakash et al.[6,5] had 35.4\% patients with T2DM duration $>10$ years and also included patients with T2DM duration $>15$ years. Our study findings are in agreement with the observations of Mobini et al.[14] who also did not find a significant association between osteoporosis and duration of diabetes. The relationship between duration of T2DM and BMD loss is often confounded with ageing. In the studies having a higher mean age of patients, the T2DM duration often coincide with the increasing age. Relatively low mean age of patients in our study could be another factor responsible for non-existence of a relationship between duration of T2DM and BMD. In a recent study, Jang et al. [ $\left.{ }^{20}\right]$ also found that T2DM males having duration of T2DM $>5$ years had low BMD as compared to those having T2DM duration $<5$ years, however, they also found that those having duration of T2DM $>5$ years were significantly older in age as compared to those having duration of diabetes $<5$ years. These findings in turn indicate that the effect of ageing as well as duration of T2DM are often coincided and must be interpreted with caution.

In our study, females had a significant association with BMD loss in T2DM. female sex in postmenopausal age group are a known risk group for osteoporosis due to significantly increased bone resorption over formation due to low levels of estrogen thus inducing accelerated bone loss [15]. Similar to our observations, Sharma et al.[6] also found BMD to be lower in females as compared to males. Studies by other workers also supported this observation [10,11].

Our study found vitamin D levels to be positively associated with BMD. There was a significant decreasing trend of mean vitamin D levels with increasing severity of BMD loss. Prevalence of vitamin D insufficiency and deficiency was also significantly higher in osteopenia and osteoporosis as compared to those with normal BMD. However, no such association was seen with serum calcium levels. The role of vitamin D in calcium resorption and bone formation is well-documented as calcium is an essential ingredient of bone. However, the association of vitamin D level and calcium with BMD in T2DM has been documented to be of differential nature in different studies. Sharma et al.[6] in their study did not find a significant correlation of both calcium and vitamin D levels with BMD. Reema et al.[11] in their study found low calcium levels to be significantly associated with BMD loss, however, they did not study the association of vitamin D levels with BMD. Kamalanathan et al.[8] in contrast did not find a significant association of either calcium or vitamin
D levels with BMD loss. Agarwal et al.[5]found low vitamin D levels to be significantly associated with an increased risk of osteopenia and osteoporosis but did not report any such association with serum calcium.

One of the limitations of present study was lack of a control group owing to which it is difficult to say whether the prevalence of osteopenia and osteoporosis is affected by T2DM status and whether the factors governing BMD loss in T2DM are different from non-diabetic individuals. Hence, further studies on a larger population with inclusion of a non-diabetic control population are recommended to understand these issues further. Secondly, we did not assess parathyroid or testosterone levels thereby missing on normocalcemic hyperparathyroidism or hypogonadism in males. Also we did not record menopausal status in female T2DM patients thereby missing in some obvious postmenopausal osteoporosis. Thirdly latest innovations in BMD include trabecular bone score(TBS) software which detects qualitative assessment of degradation of bone microarchitecture. Addition of TBS score would have helped assess T2DM patient with normal BMD who are having partially or severely degraded bone architecture. Fourthly, BTM assessment could have helped to identify role of T2DM in low BMD states.

\section{Conclusion:}

Our study had 39\% osteoporosis prevalence in T2DM (Hip:19.0\%; Spine:35.0\%) in addition to $47 \%$ osteopenia (Hip:54.0\%; Spine:43.0\%). No significant association of osteoporosis was noted with BMI, waist circumference, duration of T2DM or glycemic status. But significant association of osteoporosis was with age $\&$ female gender. Additionally, significant association of osteoporosis was observed with vitamin D deficiency or insufficiency but not with serum calcium. Prevalence of lumbar spine osteoporosis was more than hip osteoporosis but no differences among risk factors associated with overall osteoporosis as compared to lumbar spine or hip osteoporosis seperately.

\section{References:}

1. Chawla A, Chawla R, Jaggi S. Microvasular and macrovascular complications in diabetes mellitus: Distinct or continuum? Ind J Endo Metab. 2016;20(4):546-551.

2. Nyman JS, Even JL, Jo CH, et al. Increasing duration of type 1 diabetes perturbs the strength-structure relationship and increases brittleness of bone. Bone. 2011;48(4):733-740.

3. Strotmeyer ES, Cauley JA, Schwartz AV, et al. Diabetes is associated independently of body composition with BMD and bone volume in older white and black men and women: The Health, Aging, and Body Composition Study. J Bone Miner Res 2004;19(7):1084-1091.

4. Dutta M, Pakhetra R, Garg M. Evaluation of bone mineral density in type 2 diabetes mellitus patients before and after treatment. Med J Armed Forces India. 2012;68(1):48-52.

5. Agrawal NK, Sharma B. Prevalence of osteoporosis in otherwise healthy Indian males aged 50 years and above. Arch Osteoporos 2013;8:116.

6. Sharma B, Singh H, Chodhary P, Saran S, Mathur SK. Osteoporosis in otherwise healthy patients with type 2 diabetes: A prospective gender based comparative study. Ind $\mathbf{J}$ Endo Metab 2017;21:535-539.

7. Asokan AG, Jaganathan J, Philip R, Soman RR, Sebastian ST, Pullishery F. Evaluation of bone mineral density among type 2 diabetes mellitus patients in South Karnataka. J Nat Sci Biol Med. 2017;8(1):94-98.

8. Kamalanathan S, Nambiar V, Shivane V, Bandgar T, Menon P, Shah N. Bone mineral density and factors influencing it in Asian Indian population with type 2 diabetes mellitus. Ind J Endo Metab 2014;18:831-837. 
9. Sözen T, Özışık L, Başaran NÇ. An overview and management of osteoporosis. Eur J Rheumat. 2017;4(1):46-56.

10. Prakash S, Jatti RS, Ghagane SC, Jali SM, Jali MV. Prevalence of Osteoporosis in Type 2 Diabetes Mellitus Patients Using Dual Energy X-Ray Absorptiometry (DEXA) Scan. Int J Ost Metab Dis 2017;10:10-16.

11. Reema N, Jain J, Singh TG, Banait S. A study of magnitude and correlates of altered bone mineral density in type 2 diabetes mellitus patients of central rural India. Int $\mathrm{J}$ Adv Med 2020;7:1380-1387.

12. Leidig-Bruckner G, Grobholz S, Bruckner T, Scheidt-Nave C, Nawroth P, Schneider JG. Prevalence and determinants of osteoporosis in patients with type 1 and type 2 diabetes mellitus. BMC Endo Dis. 2014;14(33):1-13.

13. Thakur AK, Dash S. Estimation of bone mineral density among type 2 diabetes mellitus patients in western Odisha. Int J Res Med Sci 2018;6:459-464.

14. Mobini M, Kashi Z, Bahar A, Taslimi F, Forghanizadeh J, Abdi R. Osteoporosis and Osteoporotic Fractures in Postmenopausal Women with Type 2 Diabetes Compared with Non-Diabetic Cases. Iran J Diab Ob. 2012;4(2):68-73.
15. Demontiero O, Vidal C, Duque G. Aging and bone loss: new insights for the clinician. Ther Adv Musculoskelet Dis. 2012;4(2):61-76.

16. Cristina de Sousa E Silva Araujo E, Pagotto V, Silveira EA. Bone Mineral Density in the Noninstitutionalized Elderly: Influence of Sociodemographic and Anthropometric Factors. Curr Gerontol Geriatr Res. 2016;2016:4946593.

17. Hannan MT, Felson DT, Dawson-Hughes B, Tucker KL, Cupples LA, Wilson PW, Kiel DP. Risk factors for longitudinal bone loss in elderly men and women: the Framingham Osteoporosis Study. J Bone Miner Res. 2000;15(4):710-720.

18. Oei et al Oei L, Zillikens MC, Dehghan A, et al. High bone mineral density and fracture risk in type 2 diabetes as skeletal complications of inadequate glucose control: the Rotterdam Study. Diabetes Care. 2013;36(6):1619-1628.

19. Starup-Linde J, Gregersen S, Vestergaard P. Associations with fracture in patients with diabetes: a nested case-control study. BMJ Open 2016;6:e009686.

20. Jang et al. Jang M, Kim H, Lea S, Oh S, Kim JS, Oh B. Effect of duration of diabetes on bone mineral density: a population study on East Asian males. Endocrine Disorders: 2018;18:61.

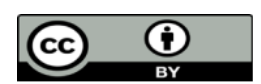

This work is licensed under Creative Commons Attribution 4.0 License

To Submit Your Article Click Here: Submit Manuscript

DOI: $10.31579 / 2641-8975 / 0028$
Ready to submit your research? Choose Auctores and benefit from:

$>$ fast, convenient online submission

$>$ rigorous peer review by experienced research in your field

$>$ rapid publication on acceptance

$>$ authors retain copyrights

$>$ unique DOI for all articles

$>$ immediate, unrestricted online access

At Auctores, research is always in progress.

Learn more https://auctoresonline.org/journals/diabetes-and-islet-biology 